\title{
The first experimental study of transference work-in teenagers (FEST-IT): a multicentre, observer- and patient-blind, randomised controlled component study
}

Randi Ulberg ${ }^{1,2,3^{*}}$ D, Benjamin Hummelen ${ }^{4}$, Anne Grete Hersoug ${ }^{1}$, Nick Midgley ${ }^{5}$, Per Andreas Høglend ${ }^{1}$ and Hanne-Sofie Johnsen Dahl ${ }^{1,6}$

\begin{abstract}
Background: Little is known about the influence on outcome of exploration of the patient-therapist relationship (that is, transference work) in psychoanalytic psychotherapy. We hypothesized that depressed adolescents would have better long-term effects from psychoanalytic psychotherapy with than without transference work.

Methods: Depressed adolescent (16 to 18 years) were recruited in health authority funded out-patient clinics in Oslo and Vestfold County, Norway. They were randomized to 28 weeks of treatment with psychoanalytic psychotherapy with or without transference work. Change was assessed using linear-mixed models. The primary outcome measure was the Psychodynamic Functioning Scale (pre- post-, and 1-year post-treatment). Level of depression was measured at the same time points and during therapy (week 12, and 20).

Results: 69 adolescents were treated with $(N=39)$ or without $(N=31)$ transference work. The mean number of sessions was $18.6(S D=8,6)$ in the transference work group and $18.0(S D=10.9)$ in the non-transference work group.

Both groups showed large and significant improvement on Psychodynamic Functioning Scale during the whole study period. The difference between the two groups was not significant during the treatment period $(95 \% \mathrm{Cl}-.79$ to $1.2, p=.674, \mathrm{~F}=.18$ ), or from post-treatment to one-year follow-up ( $95 \% \mathrm{Cl}-.13$ to $.96 ; p=.134 ; \mathrm{F}=2.3)$. For the secondary outcome measures the transference work group had significantly better outcomes from 12 weeks in treatment to one-year follow-up (Beck Depression Inventory, 95\% Cl-1.7 to -.14, $p=.022$; Montgomery and Åsberg Depression Rating Scale, 95\% Cl -1.6 to $-.23, p=.009$ ).

Conclusion: The findings suggest that exploration of the adolescents' relations to the therapist amplify the effects of short-term psychoanalytic psychotherapy on their depressive symptoms for adolescents with a Major Depressive Disorder.
\end{abstract}

\footnotetext{
* Correspondence: randi.ulberg@medisin.uio.no

'Division of Mental Health and Addiction, Institute of Clinical Medicine, University of Oslo, P.O. box 1171, 0318, Blindern, Oslo, Norway

${ }^{2}$ Vestfold Hospital Trust, Division of Mental Health, Research Unit, P.O. box 2169, 3125 Tønsberg, Norway

Full list of author information is available at the end of the article
}

(c) The Author(s). 2021 Open Access This article is licensed under a Creative Commons Attribution 4.0 International License, which permits use, sharing, adaptation, distribution and reproduction in any medium or format, as long as you give appropriate credit to the original author(s) and the source, provide a link to the Creative Commons licence, and indicate if changes were made. The images or other third party material in this article are included in the article's Creative Commons. licence, unless indicated otherwise in a credit line to the material. If material is not included in the article's Creative Commons licence and your intended use is not permitted by statutory regulation or exceeds the permitted use, you will need to obtain permission directly from the copyright holder. To view a copy of this licence, visit http://creativecommons.org/licenses/by/4.0/ The Creative Commons Public Domain Dedication waiver (http://creativecommons.org/publicdomain/zero/1.0/) applies to the data made available in this article, unless otherwise stated in a credit line to the data. 
(Continued from previous page)

Trial registration: ClinicalTrials.gov. Id: NCT01531101. Registered 8 February 2012.

Keywords: Psychodynamic, Psychoanalytic, Youth psychotherapy, Transference, Major Depressive Disorder

\section{Background}

Mental health problems, such as anxiety and depression, are estimated to affect $30 \%$ of youth worldwide. $50 \%$ of lifetime diagnosable mental health disorders start by the age of 14 , and this number increases to $75 \%$ by the age of 24. There is a need for a more comprehensive evidence base for treatment of young people with mental health problems, including depression [1].

Empirical evidence supports treatment with psychoanalytic psychotherapy in adolescents [2-4]. Short-term psychoanalytic psychotherapy has been shown to promote improvement in depressed young individuals $[4,5]$. The study "Improving mood with psychoanalytic and cognitive therapies" (IMPACT) is the most extensive trial in the field of youth depression psychotherapy [4]. In IMPACT, three manualized psychotherapy modes were compared. Short-term psychoanalytic therapy, cognitive behavioural therapy (CBT), and a brief psychosocial intervention were all found equal in terms of clinical- and cost-effectiveness. The authors reported no evidence for the superiority of any of the three treatment modes in maintenance of reduced depression symptoms approximately 12 months after treatment [4].

The IMPACT trial was not designed to establish whether the comparable outcomes were due to common factors, or whether there were distinctive features which led to comparable outcomes. There is probably an array of active ingredients in psychotherapy. However, there is a need for more knowledge on how specific psychotherapeutic techniques in the different treatment modes influence outcome over time $[3,5,6]$. The young person's experience of the therapist and the therapy is assumed to be of importance for treatment outcome [7-9]. In psychoanalytic psychotherapy the in-session exploration of the patient - therapist relationship (that is, transference work), is a key component. However one Randomized clinical trial (RCT) with adult patients, the First Experimental Study of Transference-Interpretations (FEST), found no main effects of this specific technique $[10,11]$. The present study, FEST-IT, is an adjusted replication of FEST, including adolescents with Major Depressive Disorder (MDD). The treatment is according to the treatment manual in the short-term psychoanalytic arm of IMPACT [12]. One ingredient described in the manual, the transference work, is manipulated in the two treatments in FEST-IT.

The purposes of psychoanalytic psychotherapy for MDD are reduction of depressive symptoms, but also to foster dynamic change in the young person. Positive dynamic change includes a broad spectre of areas; i.e. improved relations, increased insight in one's own reactions, enhanced tolerance for affects, and improved capacity to solve upcoming problems in life. Transference work are thought to promote dynamic change, a phenomenon which is challenging to measure. Psychodynamic Functioning Scales (PFS) [13] is a measure developed to capture evaluator-rated dynamic change. PFS has shown promising features when it comes to capture statistically and clinically significant changes in adolescents [14]. The patient's functioning during the last three months is rated based on a semi-structured dynamic interview [15]. Five sub scales relevant for rating adolescents are: Quality of Family Relations, Quality of Friendships, Tolerance for Affects, Insight, and Problem-Solving Capacity.

Building on the design of the two previous clinical trials, i.e. IMPACT and FEST, the FEST-IT trial aims to assess the effects of transference work in adolescent psychotherapy for young people with MDD. As in IMPACT, the included patients were youth with MDD. As in FEST, the patients were randomized to short-term psychoanalytic therapy with or without transference work. The primary hypothesis in FESTIT was that the transference work group would have a more favourable course than the non-transference work group over time. That is, a significant improvement during the whole study period, on the PFS, and on patient rated depression measure Beck Depression Inventory (BDI) as well as the clinician rated Montgomery Åsberg Rating Scale (MADRS). Adolescence is a time to explore and develop relational and emotional skills as well as social competency. When a depressed one withdraws from friends and family, talking about the ongoing relationship with the therapist in the here and now invites the adolescent to practice relational and emotional skills. The aim of the study was to decide whether depressed adolescents improve significantly more from short-term psychoanalytic psychotherapy with than without transference work [16], up to 1-year followup, both in terms of overall functioning (PFS), as well as depression symptoms (BDI and MADRS).

\section{Methods}

Study design and participants

Experimental Study of Transference Work-In Teenagers (FEST-IT) is a multicentre, observer- and patient-blind, randomised controlled component trial. The patients 
were included from two areas in Norway: the mainly urban areas in and around Oslo of about 1 million people, and mixed urban and rural areas in Vestfold County containing about 250,000 people. The patients were treated in outpatient clinics.

Adolescents aged 16 to 18 years, with current unipolar MDD according to Diagnostic and Statistical Manual of Mental Disorders, Fourth Edition (DSM-IV; American Psychiatric Association, 2000), were included. Adolescents with generalized learning difficulties, pervasive developmental disorder, psychosis, or substance addiction were excluded. Comorbidity was expected to be frequent. Axis I and II diagnosis were based on the Mini International Neuropsychiatric Interview (M.I.N.I.) [17] and Structured Interview for DSM-IV Personality (SIDPIV) [18]. Written consent was obtained from all patients.

\section{Randomisation and masking}

The randomisation was stratified. The allocation to treatment of patients admitted to the trial was achieved by setting up clusters of four patients for each of the 12 therapists and using lottery to allocate patients to one of the two treatment groups. For each of four patients randomised to each therapist, two patients were treated with transference work and two without. A randomisation officer with no other connection with the evaluators, therapists, study coordinator, or researchers did the randomisation. Only the therapist was aware of the randomisation, which was concealed from patients and evaluators. The randomisation key was kept by the officer and broken when the last patient had finalized the study treatment and had been evaluated at 1-year follow up.

\section{Procedure}

The 12 therapists treated patients in both treatment groups. The treatment manual developed by the IMPACT research group and used in the short-term psychoanalytic arm of IMPACT [12], was used. The patients were offered 28 sessions. The therapy mode emphasizes general psychoanalytic treatment principles, i.e. interventions exploring the young person's relationships to others, thoughts and feelings that might be avoided, as well as pointing to recurring patterns in both feelings, thoughts, and behaviour. The therapists are receptive to what the patient finds important to talk about and do not define the focus for the sessions. The patients were randomised to short-term psychoanalytic psychotherapy with either transference work or no transference work. Transference work is explained in more detail in the Supplement, Supplementary file 1: Appendix I. In the transference work group, the therapists encouraged the patients to explore their feelings and thoughts about the therapist and the therapy, as well as repetitive patterns of reactions and actions emerging during the sessions in relationship to the therapist. These interventions were offered to a moderate level (i.e. 1-3 times per session).

The therapists were experienced psychologists and psychiatrists. They had a minimum of two years of psychoanalytic training and were also trained through a one-year course with two full day seminars and monthly half day seminars based on the treatment manual [12]. During the preparation course, the focus was on the differences in the techniques when offering short-term psychoanalytic psychotherapy with or without transference work. To maintain the quality of the therapies and adherence to the manual, peer supervision groups were offered throughout the study period. This also was to ensure that the therapy mode in each therapy group was delivered. Certified supervisors in psychoanalytic psychotherapy managed the continuous training. The Principal Investigator and the study coordinator were available for the therapists at any time. The average number of attended sessions were 18.6 $(\mathrm{SD}=8.6)$ in the transference work group and 18.0 (SD = $10.9)$ in the non-transference work group. The therapists' use of the specific transference techniques differentiated significantly between the treatment groups. The level of the transference interventions were measured on a Likert scale 0-4; 2.2 (SD 1.47) in the transference work group and .52 (SD .78) in the non-transference work group ( $\mathrm{df} 52.5, \mathrm{t}=-5.5 p<0$, 0006). Intra Class Correlation among two raters (single measure) was .89 (CI $95 \% .75$ to $.96, p=.000)$.

\section{Outcomes}

The primary outcome measure was the Psychodynamic Functioning Scales (PFS). The five PFS-sub scales Quality of Family Relations, Quality of Friendships, Tolerance for Affects, Insight and Problem-Solving Capacity were used. The patients were rated with PFS by three raters blinded for randomisation at pre- and post-treatment and at 1-year follow-up. To measure the level of depression, the patients filled in a self-report scale, the BDI [19]. The MADRS [20] were rated by the therapists during therapy and by independent and blind raters at pre-, post-, and 1 year follow up. FEST-IT is a multicentre trial. Evaluators interviewed the patients and rated at pre-, post-, and 1-year follow-up. In addition to the evaluators, two experienced psychoanalysts rated all audio-taped interviews. To avoid skewness in ratings to develop, all raters were trained in scoring the PFS and MADRS and met for regular reliability ratings during the study period. The average of the evaluator ratings and the two experienced psychoanalysts' ratings of each patient interview on each time point using PFS, were used in the statistical analyses. Intra Class Correlation (ICC) for PFS was .82 (CI 0.73-0.91) [15]. MADRS was rated by one independent and blinded rater and the therapist in $30 \%$ of the cases. ICC for MADRS single measure was .78 ( $\mathrm{Cl} 0.58-0.9)$. During therapy MADRS was rated by the therapist. 


\section{Statistical analyses}

The Supplement, Supplementary file 1: Appendix II details the statistical analysis.

Based on standard t-test comparison of the two groups, 100 patients in this study would reveal a moderate effect size (0.55), with a significance level of 0.05 and a power of 0.80 . However, only 69 patients were finally included in the study. With 39 patients in the transference work group and 30 patients in the non-transference work group, power is reduced to .61. An one-sided t-test gives a power of .72, which implies that the risk of not rejecting the 0-hypothesis, i.e., it is a $28 \%$ chance that no difference in treatment outcome with respect to PFS, is not true.

\section{Linear mixed models analyses}

The mixed models procedure in SPSS (version 26) was used to investigate differences in clinical change across the two treatment groups for PFS, BDI, and MADRS (dependent variables). The components "time" and "time"treatment" were entered as independent variables/ covariates in the model. "Treatment" is a dummy variable indicating group membership, i.e., "transference treatment group" versus "non-transference treatment group". The time variable was coded by integers and each integer represents approximately 10 weeks. For PFS, time was coded as $0-3-8$, representing the three points in time when PFS was measured, i.e., pretreatment, post-treatment, and one-year follow-up. For BDI and MADRS, time was coded as $0-1-2-3-8$, representing five time points, i.e., pre-treatment, 12 weeks, 20 weeks, post-treatment, and one-year follow-up.. The interaction component "time*treatment" was included to test differential change rates across the two treatment groups. Log likelihood estimation (LLH) and Akaike Information Criterion (AIC) were used to evaluate model fit. Model parameters were estimated by maximum likelihood estimation and models were compared by log likelihood ratio tests.

The analyses were conducted in three steps (see online supplement for details). The first step aimed at modelling the mean response over time for the entire sample. The second step addressed the question whether there was differential change for the two treatment groups. The third step aimed at comparing differences in change rates across the two groups for different time periods within the same model, i.e., the "linear spline model". For PFS, the knot was placed at post-treatment in the linear spline model, which implies that the first time period was from pretreatment to post-treatment and the second period from post-treatment to one-year follow-up. For BDI and MADRS, the knot was situated at 12 weeks in treatment, which means that the first time period was from pretreatment to 12 weeks in treatment and the second period from 12 weeks to one-year follow-up. The choice of the time periods was based on visual inspection of the scatterplots including interpolation lines through mean values for each group. As part of the sensitivity analyses, the three steps were reconducted by a competing linear spline model, i.e., a model with random intercepts and random slopes at the individual level, with variance component covariance matrix for the random effects (the "random effects model").

Differences in the frequency of missing data across treatment groups were analysed be chi-square statistics, and differences in mean values of treatment outcomes were analysed by a series of ANOVAs including Tukey post hoc tests. On basis of these analyses, it was concluded that the missing data could be treated as "missing completely at random" in the current study.

The analyses were performed by an independent researcher overseen by one senior researcher at the University of Oslo and one statistician from research service at Oslo University Hospital. All 69 patients recruited from outpatient clinics included in the study were included in the intention to treat analyses. The Central Norway Regional Ethics Health Committee approved the study protocol (https://www.med.uio.no/ klinmed/english/research/projects/fest-it/pdf/fest-it protocol.pdf) (REK: 2011/1424 FEST-IT). FEST-IT is registered in ClinicalTrials.gov: NCT01531101.

\section{Role of the funding source}

The funder of the study had no role in study design, data collection, data analysis, data interpretation, or writing of the report. The corresponding author had full access to all the data in the study and had final responsibility for the decision to submit for publication.

\section{Results}

Between February 2012 and September 2017, 70 patients were randomly assigned to psychoanalytic therapy with $(N=39)$ or without $(N=31)$ transference work. 1 patient withdrew from the study. Data from 69 patients were included in the intention-to-treat analyses (Please see the flow diagram, Fig. 1).

No significant differences were observed between the transference work group and the comparison group on the pre-treatment variables (Table 1).

There was little use of medication among the patients. One patient used antidepressant medication at the beginning of therapy. One other at the end of therapy, however, not the same patient. One patient was taking antipsychotics throughout the study period. One patient at pre-treatment and 4 patients at post-treatment were taking sleeping medicine [14]. 


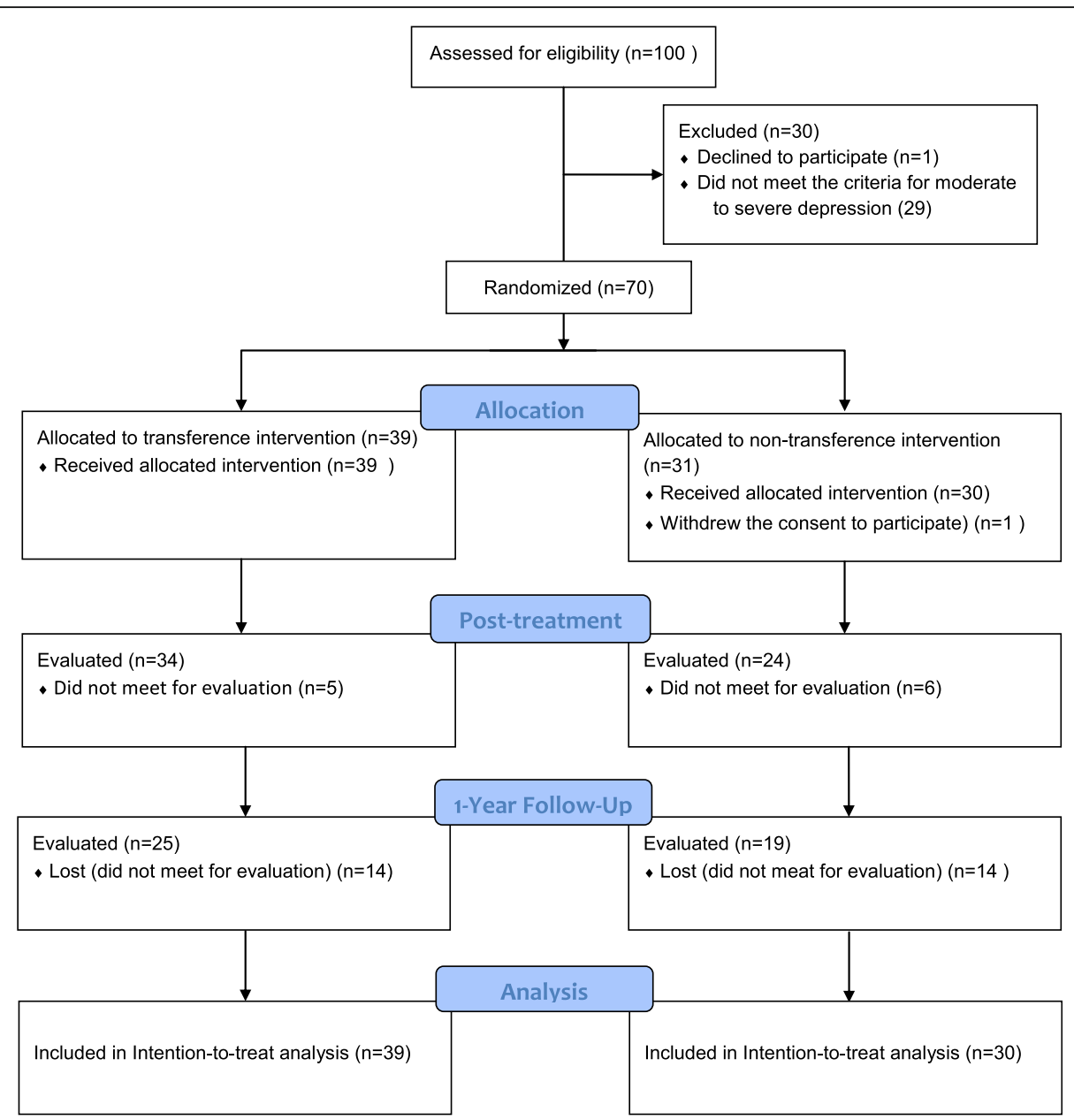

Fig. 1 Trial profile. The primary hypothesis was analyzed in 69 patients. 16 patients had missing PFS at one occasion. 16 patients had missing BDI at one occasion. 11 patients had missing MADRS at one occasion. One patient in the non-transference group withdrew consent before starting treatment

\section{Primary analyses of Outcome Variables.}

Descriptive statistics over time for the three outcome variables are presented in Table 2.

There was a significant change for PFS from pretreatment to post-treatment for the entire sample. Inspecting the first two rows of Table 3 , it can be seen that for every 10 weeks, PFS increased 1.7 points for the non-transference work group $(p=.000)$ and 1.91 points $(1.7+.21)$ for the transference work group (a nonsignificant difference; $p=.674$ ). From post-treatment to one-year follow-up, the increase of PFS was not significant for either groups $(p=.083$ for time, i.e., the nontransference work group, and $p=.134$ for time*treatment). The non-significant interactions between time and treatment indicate that the change rates did not differ significantly across the two treatment groups. However, in the linear model for PFS, the interaction between time and treatment was significant at the alpha $=.05$ level $(\mathrm{F}=4.1, p=.048)$. In this model, the non-transference work group increased .75 for every ten weeks from pre-treatment to one-year follow-up whereas the transference work group increased 1.11 points for every ten weeks. The fit of this model was significantly poorer than the fit of the spline model (see online supplement for details).

For BDI, the transference work group changed significantly during the first time period. Since the time"treatment interaction was not significant, it can be assumed that the non-transference work group also changed significantly from pre-treatment to 12 weeks (Table 3). For the second time period interaction was significant, which implies that the two groups had different change rates from 12 weeks to one-year follow-up. Inspection of the parameter estimates in Table 3 shows that the transference work group improved more than the non-transference work group during this time period (.91 points for every tenth week).

For MADRS, a similar picture was seen except that the non-transference work group did not change significantly from 12 weeks to one-year follow-up (Table 3). 
Table 1 Pre-treatment characteristics in 69 adolescents receiving 28 weeks of psychoanalytical psychotherapy with or without transference work

\begin{tabular}{|c|c|c|c|c|c|}
\hline & \multicolumn{2}{|c|}{ Transference work group $(n=39)$} & \multicolumn{2}{|c|}{ Non-transference work group $(n=30)$} & \multirow[t]{2}{*}{ Effect sizes } \\
\hline & $N$ & $\%$ & $N$ & $\%$ & \\
\hline \multicolumn{6}{|c|}{ Gender } \\
\hline Female & 33 & 84.6 & 24 & 80.0 & \\
\hline Male & 6 & 15.4 & 6 & 20.0 & $x^{2}=.25, p=.62$ \\
\hline \multicolumn{6}{|l|}{ Housing situation } \\
\hline Both parents & 15 & 38.5 & 14 & 46.6 & \\
\hline One parent or commute & 22 & 56.4 & 12 & 40.0 & $x^{2}=2.5, p=.29$ \\
\hline \multicolumn{6}{|l|}{ between two parents } \\
\hline Other & 2 & 5.1 & 3 & 10.0 & \\
\hline Missing & - & - & 1 & 3.4 & \\
\hline \multicolumn{6}{|l|}{ Diagnostics (M.I.N.I.) } \\
\hline Recurrent depression & 15 & 38.5 & 9 & 30.0 & $x^{2}=.139, p=.71$ \\
\hline Suicide risk (moderate to high) & 6 & 15.4 & 4 & 13.3 & $X^{2}=.06, p=.81$ \\
\hline Prevalence of one or more & 18 & 46.2 & 16 & 53.3 & $x^{2}=.35, p=.55$ \\
\hline \multicolumn{6}{|l|}{ comorbid diagnoses } \\
\hline & Mean & (SD) & Mean & (SD) & \\
\hline Age & 17.30 & $(0.7)$ & 17.31 & $(0.7)$ & $\mathrm{t}(66)=0.64, p=.95$ \\
\hline \multicolumn{6}{|l|}{ Personality diagnostics } \\
\hline PD criteria as measured with SIDP-IV & 13.5 & (9.0) & 12.4 & (7.8) & $\mathrm{t}(65)=-0,52, p=.60$ \\
\hline
\end{tabular}

Table 2 Outcome measures over time in 69 adolescents receiving short-term psychoanalytic psychotherapy with or without transference work

Transference work group

N Mean
Non-transference work group

Mean

(SD)

\section{Primary outcome measures}

PFS (Psychodynamic Functioning Scales)

$\begin{array}{llllllllll}\text { Pre-treatment } & 39 & 61.07 & (5.1) & 30 & 61.24 & (4.7) & 67 & .14 & .89 \\ \text { Post-treatment } & 34 & 66.94 & (6.2) & 24 & 66.79 & (6.3) & 56 & -.09 & .93 \\ \text { One-year follow-up } & 25 & 71.02 & (5.3) & 19 & 68.48 & (6.5) & 42 & -1.42 & .16\end{array}$

\section{Depression measures}

MADRS (Montgomery and Åsberg Depression Rating Scale)

\begin{tabular}{|c|c|c|c|c|c|c|c|c|}
\hline Pre-treatment & 39 & 21.67 & (6.1) & 29 & 24.14 & $(6.0)$ & 66 & 1.67 \\
\hline 12 weeks & 27 & 14.33 & (7.6) & 19 & 13.58 & (7.3) & 44 & -.34 \\
\hline 20 weeks & 21 & 13.62 & (5.9) & 15 & 13.73 & $(7.4)$ & 34 & .52 \\
\hline Post-treatment & 33 & 12.76 & (7.7) & 25 & 11.90 & $(7.5)$ & 56 & -.43 \\
\hline One-year follow-up & 23 & 7.09 & (7.0) & 20 & 12.28 & $(8.0)$ & 41 & 2.26 \\
\hline
\end{tabular}

BDI (Becks Depression Inventory)

\begin{tabular}{llllllllll} 
Pre-treatment & 35 & 28.24 & $(9.7)$ & 28 & 29.10 & $(8.4)$ & 61 & .39 & .71 \\
12 weeks & 28 & 21.66 & $(11.7)$ & 19 & 20.38 & $(11.7)$ & 45 & -.37 & .71 \\
20 weeks & 21 & 19.04 & $(12.4)$ & 15 & 20.57 & $(13.2)$ & 34 & .36 & .72 \\
Post-treatment & 34 & 15.45 & $(11.6)$ & 24 & 17.21 & $(14.3)$ & 56 & .52 & .61 \\
One-year follow-up & 26 & 8.42 & $(10.9)$ & 20 & 14.75 & $(11.9)$ & 44 & 1.88 & .07 \\
\hline
\end{tabular}


Table 3 Results from linear spline models for Psychodynamic Functioning Scales, Beck Depression Inventory, and Montgomery and Åsberg Depression Rating Scale

\begin{tabular}{|c|c|c|c|c|c|c|c|}
\hline Dependent variable & Estimate & SE & $\mathrm{Cl}(95 \%)$ & Df & F-value & $\mathbf{t}$ & $p$ \\
\hline \multicolumn{8}{|c|}{ Psychodynamic functioning scale } \\
\hline \multicolumn{8}{|c|}{ From pre- to post-treatment } \\
\hline Time $^{a}$ & 1.7 & .39 & .92 to 2.5 & 62.0 & 46.5 & 4.3 & .000 \\
\hline Time $\mathrm{x}$ treatment ${ }^{\mathrm{b}}$ & .21 & .50 & -.79 to 1.2 & 58.1 & .18 & .67 & .674 \\
\hline \multicolumn{8}{|c|}{ Post-treatment to one-year follow-up } \\
\hline Time $^{a}$ & .37 & .21 & -.05 to .78 & 47.3 & 17.8 & 1.8 & .083 \\
\hline Time $x$ treatment ${ }^{b}$ & .41 & .27 & - . .13 to .96 & 47.0 & 2.3 & 1.5 & .134 \\
\hline \multicolumn{8}{|c|}{ Beck Depression Inventory } \\
\hline \multicolumn{8}{|c|}{ From pre-treatment to 12 weeks } \\
\hline Time $^{a}$ & -10.1 & 2.0 & -14.0 to -6.1 & 65.1 & 43.8 & -5.1 & .000 \\
\hline Time $\mathrm{x}$ treatment ${ }^{\mathrm{b}}$ & 2.8 & 2.5 & -2.2 to 7.9 & 61.6 & 1.2 & 1.1 & .270 \\
\hline \multicolumn{8}{|c|}{ From 12 weeks to one-year follow-up } \\
\hline Time $^{a}$ & -.83 & .30 & -1.4 to -.23 & 55.9 & 39.4 & -2.8 & .008 \\
\hline Time $\mathrm{x}$ treatment ${ }^{\mathrm{b}}$ & -.91 & .38 & -1.7 to -.14 & 48.6 & 5.6 & 2.4 & .022 \\
\hline \multicolumn{8}{|l|}{ MADRS } \\
\hline \multicolumn{8}{|c|}{ From pre-treatment to 12 weeks } \\
\hline Time $^{a}$ & -9.5 & 15 & -12.5 to -6.5 & 59.7 & 60.4 & -6.3 & .000 \\
\hline Time $x$ treatment ${ }^{b}$ & 1.9 & 1.8 & -1.7 to 5.5 & 49.6 & 1.1 & -1.0 & .390 \\
\hline \multicolumn{8}{|c|}{ From 12 weeks to one-year follow-up } \\
\hline Time $^{a}$ & -.22 & .25 & -.72 to .29 & 49.6 & 15.8 & -.87 & .302 \\
\hline Time $\mathrm{x}$ treatment ${ }^{\mathrm{b}}$ & -.91 & .33 & 1.6 to .23 & 45.8 & 7.4 & 2.7 & .009 \\
\hline
\end{tabular}

Note: a. Change rate for the non-transference work group; b. Difference in change rates for the two treatment groups. For instance, during the second time period (from 12 weeks into treatment to one-year follow-up), BDI for the non-transference work group decreased by .83 points for every 10 weeks, and BDI for the transference work group had decreased by 1.74 points for every ten weeks $(.83+.91$ points)

The transference work group improved significantly more than the non-transference work group (Fig. 2).

\section{Discussion}

The present study had a dismantling design in which a single component in an existing and manualized treatment method (short-term psychoanalytic therapy) was varied. The efficacy of a specific technique (transference work) was investigated. On average, patients in both treatment groups showed a significant and large improvement measured with PFS, however no outcome differences between treatment groups. The level of depression decreased significantly more among the depressed adolescents in the transference work than the non-transference work group from mid-therapy (session 12) to 1 year post therapy when measured with the patient-rated BDI and the therapist- and evaluator rated MADRS. When the therapists encouraged adolescents to discuss their thoughts and feelings towards the therapist in the here and now (transference work), the young person improved significantly more on symptom measures.

The need for elaborating the patient-therapist relationship with adolescents in therapy is underlined [7, 21].
The treatment fidelity statistics show us that the dialogue focusing on the therapeutic relationship is present to a moderate extent in the transference work therapies, but absent from the non-transference therapy sessions, where this is not initiated by the therapist. Hence, adolescents, like the adults in the FEST study [22], do not by themselves discuss the therapeutic relationship or alliance, they need to be invited to do so. Then, the exploration of oneself in relation to the therapist, may comprise a balance between autonomy and acknowledgment, as well acceptance of oneself, which would be of importance for relieving depressive symptoms. Løvgren and colleagues [23] interviewed adolescents after transference work and non-transference work therapies, they found that the depressed adolescents reported the importance of the patient-therapist relation as helpful, characterised by an experience of confidence and trust in a supportive therapist. Could it be, that this experience is enhanced when the therapist invites the youngster to explore their feelings, including the negative ones, towards the therapist? Within psychodynamic theory, one aspect of depression is thought to be misplaced aggression directed inwards [12]. 


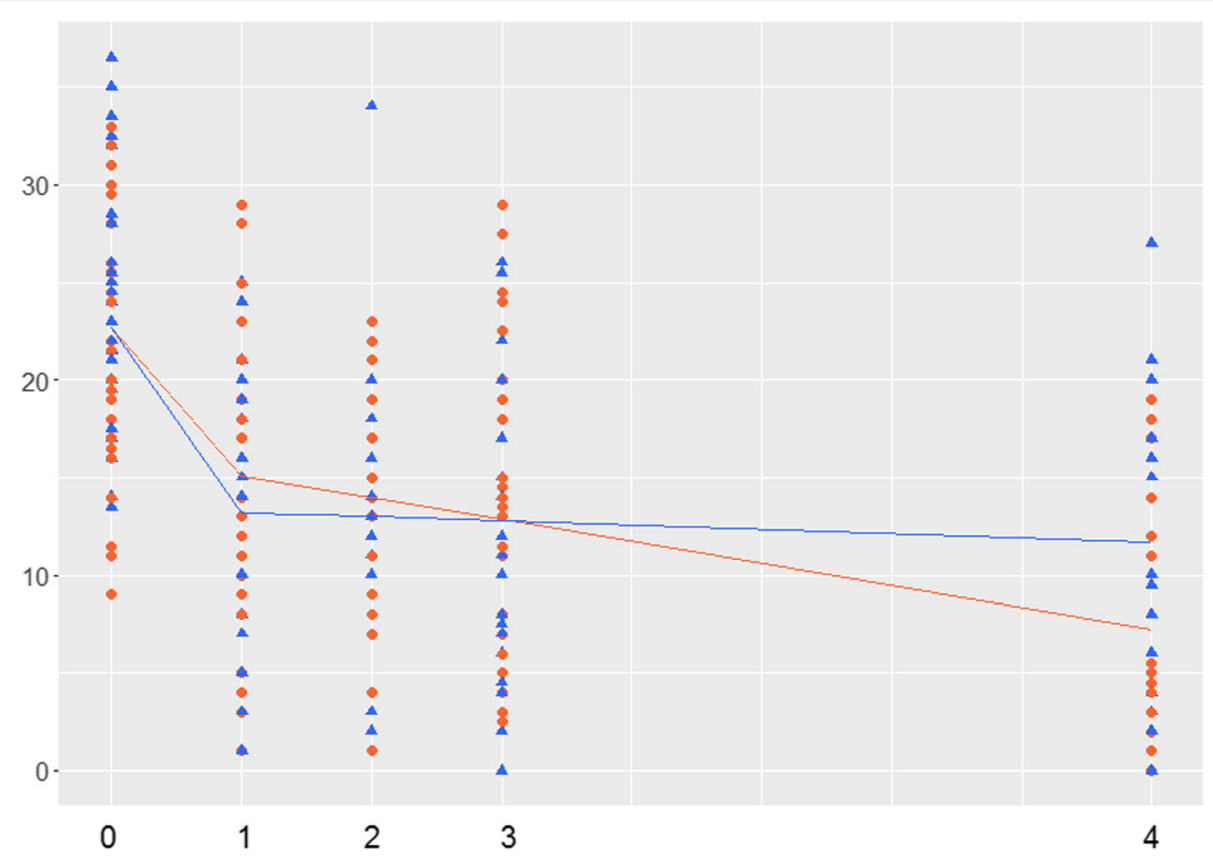

Fig. 2 Mean trajectories for Montgomery and Åsberg Depression Rating Scale (MADRS) over time for patients in psychotherapy with or without transference interventions. The level of MADRS over time. 0, pre-treatment; 1 and 2, measures during therapy; 20 weeks; 3, post-treatment; 4, post-treatment

When the therapist invites the adolescent to criticize, express annoyance or frustration directed towards the therapist, it might assist to identify aggressive feelings that are not only directed towards self. This would be expected to reduce the depressive hold on the adolescent mind. According to Løvgren and colleagues, working with the relation to the therapist in the here and now also gave the adolescents an opportunity to explore the ongoing relationship, helping them to separate their inner world from outer reality something which is diluted while depressed, and to practice relational skills. Both may precede the reduction in depressive symptoms observed [23].

A central finding was that for depressive symptoms, differential change largely occurred at post-treatment. This effect seems also to be present for PFS but in a lesser degree and not significant at the alpha $=.05$ level. This non-significant finding might be due to a type II error, underlining the need for replication. Hundred patients were sought for the study, yet only 69 were included due to time limit and economic resources. In addition, not all adolescents came for follow up evaluations, $84 \%$ came for post-treatment evaluation and $64 \%$ attended one-year evaluation. We do not know if there are systematic variations in the group of adolescents that did not show, but the missing data analyses did not show any systemic variations (see Supplement). Only two patients received antidepressant medication. No adolescents from minority populations were referred to the study, and few boys were also referred, hence there are generalizability issues. The absence of a notreatment control group restricts the assertion that the treatments were causally effective.

In their discussion of the IMPACT study, Asarnow and Ougrin [24] concluded that there was a need for more research aimed at "understanding key elements and mechanisms contributing to treatment effectiveness". As an RCT investigating the effects of one single therapist technique, FEST-IT is in line with this research challenge. However, for therapists to be supported by empirical results when adjusting and tailoring their treatment approaches to the individual patient, more research on personalized treatment is needed. Still we do not know empirically why or for whom transference work is of significance in the depressed adolescent population. Increased knowledge is needed about what treatment works best for whom and what factors in the individual patient interact with the techniques of treatments, for better or for worse [25].

\section{Conclusion}

There is a need to strengthen empirically support for which techniques are critical to improve the level of depressive symptoms in adolescents with MDD. Results from the main analyses in FEST-IT indicates that if the therapists for a specific age group of adolescent (16-18 years) focus on the patient-therapist relationship, explore 
the patient's thoughts and feelings about the therapist, and negotiate the relationship, then this increases the treatment effects on depressive symptoms in the therapy.

\section{Supplementary Information}

The online version contains supplementary material available at https://doi. org/10.1186/s12888-021-03055-y.

\section{Additional file 1.}

\section{Abbreviations}

BDI: Beck Depression Inventory; CBT: Cognitive Behavioural Therapy; DSMIV: Diagnostic and Statistical Manual of Mental Disorders, Fourth Edition; FEST: First Experimental Study of Transference-Interpretations; FEST-IT: First Experimental Study of Transference Work-In Teenagers; IMPACT: Improving mood with psychoanalytic and cognitive therapies"; MDD: Major Depressive Disorder; M.I.N.I.: Mini International Neuropsychiatric Interview; MADR S: Montgomery and Åsberg Depression Rating Scale; PFS: Psychodynamic Functioning Scales; SIDP-IV: Structured Interview for DSM-IV Personality

\section{Acknowledgments}

Thank you to all patients, therapists, and evaluators participating in this clinical trial; Svein Amlo for extensive support, clinical and theoretical supervision, rating of data and sharing research experience; Yngve Borgen Austad and Sigrun Borgen Austad for data management; Jens Egeland for supervising in data management; Peter Fonagy for participating in designing of the study; Ole Klungsøyr for overseeing the data analyses; and Peter Tallberg for rating of data.

\section{Authors' contributors}

$\mathrm{RU}, \mathrm{AGH}$, and $\mathrm{PAH}$ were responsible for drafting the original protocol. $\mathrm{RU}$ as chief investigator was securing funding, for the trial, and had overall responsibility for the management of the study. She also participated in the coordination of the study. HSJD was the study coordinator who coordinated evaluations and randomisation. RU and AGH provided training and supervision for the therapists. RU coordinated the database, with all data held in a single repository on the University of Oslo. RU and HSJD coordinated and supervised the treatment fidelity project and analysed the data with $\mathrm{BH}$ and $\mathrm{PAH} . \mathrm{BH}, \mathrm{RU}$, and HSJD wrote the statistical analysis plan and did the analyses. HSJD and RU were responsible for data cleaning. RU wrote the initial draft of the manuscript. All authors contributed to and approved the final manuscript

\section{Funding}

The FEST-IT study was funded by grants from Vestfold Hospital Trust, The University of Oslo, Oslo University Hospital, The MRK Foundation and Josef and Haldis Andresens Legat also supported the study.

\section{Availability of data and materials}

The data set used in this article is available in anonymised version on request from the first author.

\section{Ethics approval and consent to participate}

Regional Committees for Medical and Health Research Ethics in Norway approved the study protocol and the information given to the patients (REC Central, 2011/1424). Written informed consent to participate in this trial was obtained from each participant, aged 16-18 years at inclusion. According to Norwegian legislation, ethical consent for participation in research may be given from age 16 .

\section{Consent for publication}

Please see the previous paragraph

\section{Competing interests}

The authors declare that they have no competing interests.

\section{Author details}

'Division of Mental Health and Addiction, Institute of Clinical Medicine, University of Oslo, P.O. box 1171, 0318, Blindern, Oslo, Norway. ${ }^{2}$ Vestfold Hospital Trust, Division of Mental Health, Research Unit, P.O. box 2169, 3125 Tønsberg, Norway. ${ }^{3}$ Department of Psychiatry, Diakonhjemmet Hospital, Box 85 Vinderen 0319, Oslo, Norway. ${ }^{4}$ Division of Mental Health and Addiction, Oslo University Hospital, P.O. box 4959, N-0424, Nydalen, Oslo, Norway. ${ }^{5}$ University College London (UCL) and Anna Freud National Centre for Children and Families, London, UK. ${ }^{6}$ Department of Psychology, Faculty of Social Sciences, University of Oslo, Postboks 1094 Blindern, 0317 Oslo, Norway.

Received: 7 September 2020 Accepted: 13 January 2021 Published online: 17 February 2021

\section{References}

1. Patton GC, Sawyer SM, Santelli JS, et al. Our future: a lancet commission on adolescent health and wellbeing. Lancet. 2016;387:2423-78.

2. Midgley N, Kennedy E. Psychodynamic psychotherapy for children and adolescents: a critical review of the evidence base. J Child Psychother. 2011; 37:232-60.

3. Midgley N, O'Keeffe S, French L, Kennedy E. Psychodynamic psychotherapy for children and adolescents: an updated narrative review of the evidence base. J Child Psychother. 2017:43:307-29.

4. Goodyer IM, Reynolds S, Barrett B, et al. Cognitive behavioural therapy and short-term psychoanalytical psychotherapy versus a brief psychosocial intervention in adolescents with unipolar major depressive disorder (IMPA CT): a multicentre, pragmatic, observer-blind, randomised controlled superiority trial. Lancet Psychiat. 2016:4:109-19.

5. Trowell J, Joffe I, Campbell J, et al. Childhood depression: a place for psychotherapy. An outcome study comparing individual psychodynamic psychotherapy and family therapy. Eur Child Adolesc Psychiatry. 2007;16: 157-67.

6. Midgley N, Cregeen S, Hughes C, Rustin M. Psychodynamic psychotherapy as treatment for depression in adolescence. Child Adolesc Psychiatr Clin N Am. 2013;22:67-82.

7. Davidson M "You have to have the relationship": a youth perspective on psychotherapy and the development of a therapeutic relationship: Burnaby, Arts \& Social Sciences: Department of Psychology, Simon Fraser University ; 2012.

8. Della Rosa E, Midgley N. Adolescent patients' responses to interpretations focused on endings in short-term psychoanalytic psychotherapy. J Infant Child Adolesc Psychother. 2017;16:279-90.

9. Henriksen AK. Helpful aspects at the beginning of successful outpatient treatment: retrospective views of adolescents and their therapists. J Psychother Integr. 2017;27(4):508.

10. Høglend P, Bogwald KP, Amlo S, et al. Transference interpretations in dynamic psychotherapy: do they really yield sustained effects? Am J Psychiatry. 2008;165:763-71.

11. Ulberg R, Høglend P, Marble A, Sørbye $\varnothing$. From submission to autonomy: approaching independent decision making. A single-case study in a randomized, controlled study of long-term effects of dynamic psychotherapy. Am J Psychother. 2009;63:227-43.

12. Catty J, Cregeen S, Hughes C, Midgley N, Rhode M, Rustin M. Short-term psychoanalytic psychotherapy for adolescents with depression: a treatment manual. The Tavistock clinic series / the developments in psychoanalysis series. London: Karnac; 2017.

13. Høglend P, Bøgwald K-P, Amlo S, et al. Assessment of change in dynamic psychotherapy. J Psychother Pract Res. 2000;9:190.

14. Ness E, Dahl H-SJ, Tallberg P, et al. Assessment of dynamic change in psychotherapy with asdolescents. Child Adolesc Psychiatry Mental Health. 2018;12:39.

15. Ulberg R, Dahl H-SJ, Amlo S, Blayvas P, Høglend P. Psychodynamic assessment of youth and adults unsing semistructured interview and function scales (Norwegian: Psykodynamisk kartlegging av ungdom og voksne ved hjelp av semistrukturert intervju og funksjonsskalaer). Mellanrummet. 2018;36:29-31.

16. Ulberg R, Hersoug AG, Hoglend P. Treatment of adolescents with depression: the effect of transference interventions in a randomized controlled study of dynamic psychotherapy. Trials. 2012;13:159. 
17. Sheehan DV, Lecrubier Y, Sheehan KH, et al. The MINI-international neuropsychiatric interview (MINI): the development and validation of a structured diagnostic psychiatric interview for DSM-IV and ICD-10. 1998.

18. Jane JS, Pagan JL, Turkheimer E, Fiedler ER, Oltmanns TFJCP. The interrater reliability of the Structured Interview for DSM-IV Personality. 2006;47:368-75.

19. Beck AT, Steer RA, Ball R, Ranieri WF. Comparison of Beck depression inventories-IA and-II in psychiatric outpatients. J Pers Assess. 1996;67:588-97.

20. Svanborg P, MJJoad A. A comparison between the Beck depression inventory (BDI) and the self-rating version of the Montgomery Åsberg depression rating scale (MADRS). J Affect Disord. 2001;64:203-16.

21. Gergov VKH, Marttunen M, Lipsanen J, Tainio V-M, Lindberg N. Subjective outcomes of psychotherapeutic interventions: a naturalistic follow-up study among Finnish adolescent psychiatric out-patients. Psychiatr Fenn. 2015;46: 85-106.

22. Høglend P, Amlo S, Marble A, et al. Analysis of the patient-therapist relationship in dynamic psychotherapy: an experimental study of transference interpretations. Am J Psychiatry. 2006;163:1739-46.

23. Løvgren A, Røssberg Jl, Nilsen L, Engebretsen E, RJBP U. How do adolescents with depression experience improvement in psychodynamic psychotherapy? A qualitative study. BMC Psychiatry. 2019;19:95.

24. Asarnow J, Ougrin D. From efficacy to pragmatic trials: does the dodo bird verdict apply? Lancet Psychiat. 2016;4:84-5.

25. Aitken M, Haltigan JD, Szatmari P, Dubicka B, Fonagy P, Kelvin R, et al. Toward precision therapeutics: general and specific factors differentiate symptom change in depressed adolescents. J Child Psychol Psychiatry. 2020;61:998-1008.

\section{Publisher's Note}

Springer Nature remains neutral with regard to jurisdictional claims in published maps and institutional affiliations.

Ready to submit your research? Choose BMC and benefit from:

- fast, convenient online submission

- thorough peer review by experienced researchers in your field

- rapid publication on acceptance

- support for research data, including large and complex data types

- gold Open Access which fosters wider collaboration and increased citations

- maximum visibility for your research: over $100 \mathrm{M}$ website views per year

At $\mathrm{BMC}$, research is always in progress.

Learn more biomedcentral.com/submissions 